\title{
Prevalence of treated autism spectrum disorders in Aruba
}

\author{
Ingrid D. C. van Balkom • Michaeline Bresnahan • \\ Marrit F. Vogtländer • Daphne van Hoeken • \\ Ruud B. Minderaa • Ezra Susser · Hans W. Hoek
}

Received: 16 September 2008 / Accepted: 19 March 2009 /Published online: 14 April 2009

(C) Springer Science + Business Media, LLC 2009

\begin{abstract}
To study autism outside of a narrow range of settings previously studied, and in a particularly distinctive setting in the Caribbean. The aim of the Aruba Autism Project was to determine the prevalence of autism spectrum
\end{abstract}

Significant Outcomes:

-This first epidemiologic study on ASD in the Caribbean shows prevalence estimates and gender distribution similar to those reported in recent studies in the UK and US.

- Comparison analysis with a study of cumulative incidence of $\mathrm{AD}$ and ASD in the UK showed a cumulative incidence rate in the youngest age group in Aruba at the high end of the confidence interval of that in the UK.

Limitations:

- Only children who presented for clinical assessment and elicited clinical suspicion of falling within the autism spectrum are included in the prevalence estimate.

- Cases born in the early 1990s may have emigrated in search of services prior to the clinic's opening.

-Higher functioning cases born in the late 1990s may not have attained a sufficient age for referral.

I. D. C. van Balkom

Child and Adolescent Psychiatry Clinic,

Oranjestad, Aruba

M. Bresnahan • E. Susser $\cdot$ H. W. Hoek Mailman School of Public Health of Columbia University, New York, USA

M. Bresnahan • E. Susser

New York State Psychiatric Institute,

New York, USA

M. F. Vogtländer · D. van Hoeken · H. W. Hoek

Parnassia Bavo Psychiatric Institute,

The Hague, The Netherlands disorders (ASDs) in birth years 1990-1999 in Aruba. A record review study was conducted; cases were ascertained from children treated at the Child \& Adolescent Psychiatry Clinic of Aruba, the first and only child psychiatry service on the island. In these 10 birth years we found a prevalence for autistic disorder (AD) of 1.9 per 1,000 (95\% CI 1.2-2.8) and for autism spectrum disorders of 5.3 per 1,000 (95\% CI 4.1-6.7). Comparison analysis with a cumulative incidence report from the UK, showed a similar cumulative incidence to age five in Aruba. Prevalence of ASDs in birth years 1990-1999 and cumulative incidence to age five in Aruba are similar to recent reports from the United Kingdom and the United States.

Keywords Autism · Epidemiologic study · Prevalence · Cross-cultural

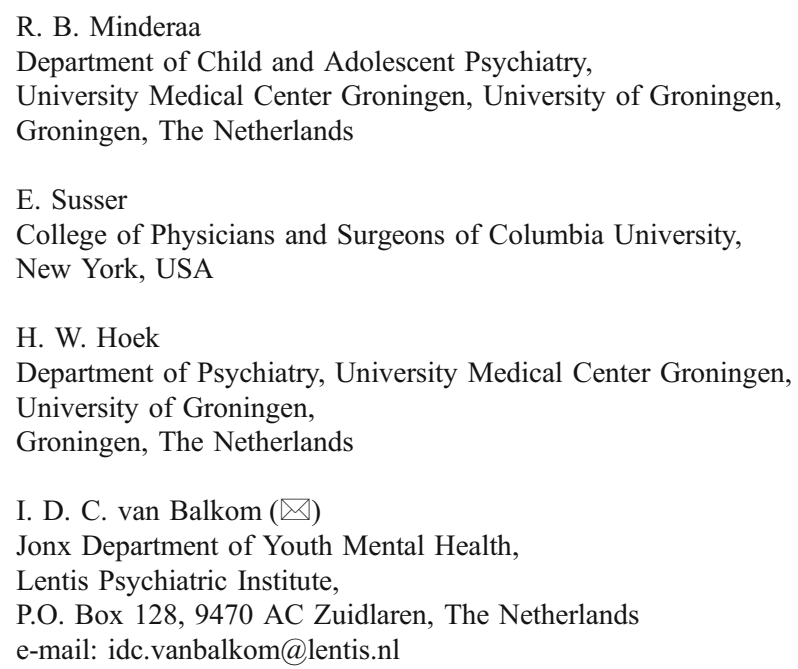




\section{Introduction}

Autism spectrum disorders (ASDs) are developmental disorders, characterized by impairments in social functioning, communication and behavior. Reports of worldwide prevalence figures since the 1990's contributed to intensified research efforts $[1,2]$, but the descriptive epidemiology of ASDs remained incomplete, as concern over potential environmental causes continued to increase [3].

For decades available data derived from prevalence studies in developed countries conducted in a narrow range of settings [1]. Current prevalence estimates of ASDs in these settings fall in the range of 3-12 per 1,000 [4-8]. Autism specific epidemiologic research outside of the narrow range of these first world high-income countries has only recently been addressed [9-11]. The current project contributes to the expansion of autism specific epidemiologic research in increasingly diverse settings.

This study is the first attempt to study the epidemiology of ASDs in the Caribbean, using methods that allow comparisons with other studies. Aruba is uniquely suited for this purpose, as it has a culturally distinct, heterogeneous, multilingual and ethnically mixed population.

Furthermore, Aruba has a well-established health care system, one centralized child psychiatry clinic and a population registry providing both the means to identify disabled children, and to enumerate the island population.

Previous psychiatric epidemiologic research in rare disorders in the same region (the Netherlands Antilles) has shown that such research is feasible $[12,13]$.

Aims of the study were to determine the prevalence for ASDs in birth years 1990-1999 in Aruba, and to conduct a comparison analysis with a cumulative incidence report from the UK. We performed the study because examining prevalence of autism across diverse settings might provide clues to either genetic or environmental etiologies.

\section{Material and methods}

Area and population

Aruba, a Caribbean island 17 miles off the coast of Venezuela, is a separate, autonomous member of the Kingdom of the Netherlands. Since 1990, the population of Aruba increased nearly $37 \%$ through immigration to 90,506 inhabitants in 2000. The population of Aruba is predominantly of Amerindian (Arawak), Dutch, and Spanish ancestry [14]. While there may be social distinctions based on race, these are nowhere documented, and race is officially considered a continuously distributed trait.

In some ways Aruban health characteristics such as life expectancy, leading causes of death, and infant mortality are similar to those of the UK, the Netherlands, and the US [15-17].

During the 1990's health insurance was nearly universal for legal residents, although access to specialty services was limited. After the introduction of a mandatory universal health insurance system in 2001 for legal residents, access to health care in Aruba improved further. Children are entitled to health care based on legal residency of parents. The insurance premium is income-dependent up to a certain maximum and paid by employers, employees and the government.

\section{Study population}

The Aruba Autism Project was carried out at the Child and Adolescent Psychiatry (CAP) Clinic of Aruba, an outpatient clinic established in 1997. The clinic was the first and only child psychiatry service on the island. Before its establishment no child psychiatry expertise or services were locally available to the community. Children could be referred to the service by general practitioners, pediatricians or other medical specialists. Virtually all Aruban-born children within the psychiatry service were legal residents.

This study is based on children born in Aruba from January 1, 1990 through December 31, 1999 and seen at the Child and Adolescent Psychiatry Clinic between May 1, 1997 and December 31, 2003.

All clinical files for the period from the establishment of the clinic from May 1, 1997 until December 31, 2003 were reviewed for the study. In this six and a half year period the clinic evaluated a total of 1,543 patients, including 813 Aruban children born in the targeted years (see Fig. 1).

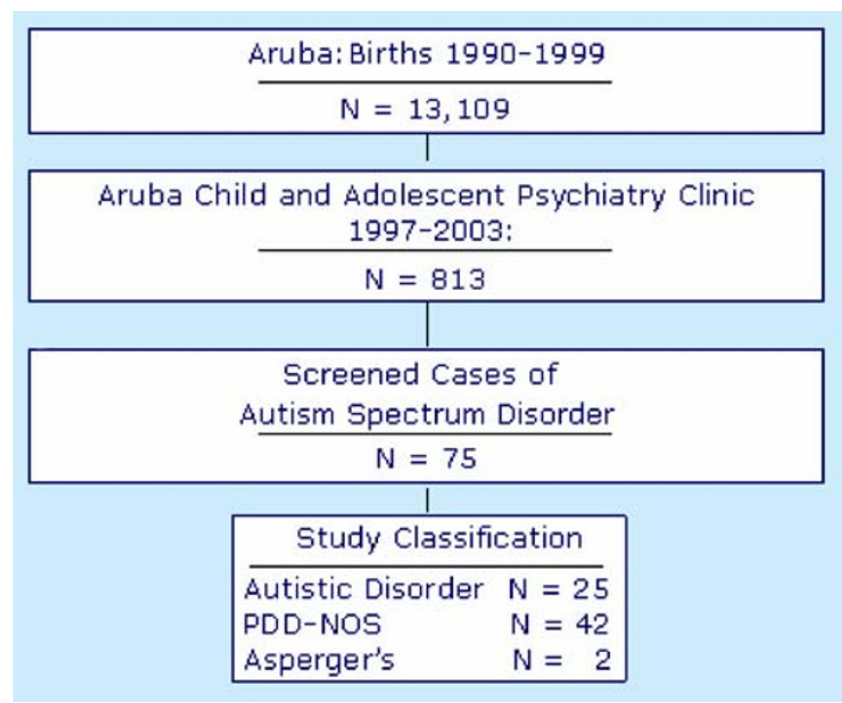

Fig. 1 Study sample 
Case ascertainment

Clinic charts of children born in the targeted birth years $(N=813)$ were systematically screened, and the records of potential cases abstracted $(N=75)$. Study diagnoses were assigned based on abstracted data.

\section{Clinical records}

Clinical notes and DSM-IV symptoms were recorded by the one clinic child psychiatrist (IvB) over the entire study period. The clinic psychiatrist was trained as a child psychiatrist in the Netherlands, where she did residencies in pediatrics, genetics, psychiatry and child psychiatry. She has expertise in developmental and genetic syndromes [1820], and is certified in the use of standardized assessment instruments for research diagnosis of ASDs. Standardized forms were completed on every child at intake. Intake included collection of medical, developmental, school and family histories; as well as information on current clinical symptoms. Diagnostic assessments were rarely concluded in one visit. Child psychiatric assessments and behavioral observations would typically take place at second and third follow up visits. Results of routine psychological assessments performed at intake or during the course of treatment were also recorded, as were notes from consultations with other experts.

At the clinic a 'suspected' ASD diagnosis was given when clinical symptoms did not (yet) meet all the criteria necessary for a definite diagnosis of a pervasive developmental disorder. A 'suspected' diagnosis made support and intervention possible, while allowing parents time to adjust, and the symptomatology to fully emerge over time [21].

For the study the clinical cases with a 'suspected' ASD diagnosis were subject to review and not automatically included in the numerator of prevalence figures. If a suspected case was assigned an ASD study diagnosisbased on the presence of DSM symptoms in the chart notes-then he/she was included as a case in the prevalence figure.

Charts with an ASD diagnosis (Autistic Disorder, Asperger's Disorder, Pervasive Developmental Disorder Not Otherwise Specified, Rett's Disorder, or Childhood Disintegrative Disorder), or an ASD diagnosis "suspected" with no subsequent diagnosis ruling out spectrum diagnoses, were flagged and abstracted by a Dutch resident of psychiatry (MV).

\section{Record review and study diagnosis}

Evidence of DSM-IV symptoms was abstracted from the charts; subsequently a computer algorithm was applied. The algorithm was consistent with DSM-IV symptom list ratings for ASDs. Study inclusion was based on standardized chart abstractions; a study diagnosis was assigned in accordance with standardized diagnostic criteria.

A study diagnosis of Autistic Disorder (AD) required chart evidence of two or more social criteria, one or more communication criteria, and one or more behavioral criteria for autism, totaling six or more criteria across the three symptom domains, with a delay or abnormal functioning onset before age three in at least one of three areas - social interaction, language used in social communication, symbolic or imaginative play. The Asperger's Disorder (AS) classification required chart evidence of two or more social criteria for autism, one or more behavioral criteria for autism, normal language development (single words by 24 months, phrase speech by 36 months), and absence of mental retardation and autistic disorder. Pervasive Developmental Disorder-Not Otherwise Specified (PDD-NOS) classification required one or more social criteria, and one or more communication criteria for autism or one or more behavioral criteria for autism in the absence of other diagnosable autism spectrum disorders. An Autism Spectrum Disorder (ASD) classification was also applied to those classified with AD, AS or PDD-NOS.

\section{Mental retardation}

Mental retardation (MR) was diagnosed based on chart records of IQ measurements. When children were unequivocally MR $(N=15)$, they received a clinical diagnosis of MR and were not tested (22\%). Measurements were available in 54 of the study diagnosed cases (78\%): in 40 cases the children had a normal test-based IQ, while 14 children $(20 \%)$ had been tested as mentally retarded (IQ $<$ 70). In total 29 of the 69 cases were mentally retarded $(42 \%)$.

\section{Validation study}

To validate study classification of ASD, the Autism Diagnostic Observation Schedule (ADOS) was used. The ADOS is a semi-structured, standardized assessment of communication, social interaction and imaginative play for individuals suspected of having an Autism Spectrum Disorder [22, 23].

The subjects included 24 children with a study diagnosis of ASD, and six clinic children diagnosed with other disorders. Selection of non-ASD clinic children was based on random identification in the computerized patient list. This list was restricted to active patients, excluding children who had previously screened positive for our study. To prevent possible selection bias the secretary of the clinic invited parents from a list of children with regular followup visits to the clinic in preceding weeks, working down 
the list until six children were selected. The selected children were born 1993-1999.

Most subjects in the validation study were assessed by an independent ADOS certified rater from the neighboring island of Curaçao. When this rater was not available, children were assessed by the first author.

\section{Confidentiality}

In keeping with Dutch medical ethical guidelines for the conduct of record review studies, personal information was treated confidentially. Only the treating child psychiatrist and the research psychiatrist had access to the medical charts. Data were entered into a statistical database without identifying information.

Population data

Data on registered births and population characteristics were obtained from the Central Bureau of Statistics, Aruba. All births to legal residents are registered. During the years 1990-1999 there were 13,109 registered births $(6,755$ males and 6,354 females) [24].

Analysis

\section{Main prevalence analysis}

Prevalence estimates were calculated as the number of cases identified among children born in Aruba between 1990-1999 divided by total registered live births 19901999. We used the Poisson distribution to calculate $95 \%$ confidence intervals [25].

Statistical comparison of the proportion with mental retardation among the diagnostic groups (AD versus PDDNOS) was assessed with Pearson Chi Square.

\section{Comparison analysis of cumulative incidence}

The published study most similar to the Aruba study in methodology and birth years was conducted by Powell et al. 2000 [26] in two areas of the West Midlands, UK. To compare our data to those described by Powell et al., 2000, we calculated cumulative incidence of AD and ASDs in children with first service contact prior to age five, and born in the period January 1, 1996 through December 31, 1998. Children in these birth years had access to the psychiatric service at least from ages one through 5 years. The cumulative incidence is calculated by the number of Aruban born children 1996-1998 with an intake prior to age five assigned a study diagnosis of ASD, divided by the total number of children born in Aruba between 19960-1998.

\section{Results}

Characteristics of the screened positive children

A total of 813 children born in Aruba from 1990 through 1999 were identified in the Child and Adolescent Psychiatry Clinic records; of these 75 children screened positive.

Among the screen positive $60 \%$ of children were referred by GP's, $30.7 \%$ were referred by pediatricians and $9.3 \%$ by other specialists. The most common reasons for referral were behavioral problems $(61.3 \%$ of children) and speech and/or language delay (45.3\% of children). Other reasons for referral were impaired relatedness $(34.7 \%)$, mental retardation (4\%), attentional problems, eating problems, sleeping disorder, or anxiety. In total $54.6 \%$ of children had more than one reason for referral.

Main prevalence findings

Sixty-nine of the screen positive children were assigned a study classification of ASD. $25(36.2 \%)$ were classified as $\mathrm{AD}, 2(2.9 \%)$ were classified as AS, and 42 (60.9\%) were classified as PDD-NOS. No cases with regression in development, Childhood Disintegrative Disorder or Rett's Disorder were identified. The overall prevalence of ASDs was 5.3 (95\% CI 4.1-6.7) per 1,000 births. Details concerning prevalence are shown in Table 1.

Case characteristics

Clinical characteristics of the 69 cases are presented in Table 2. The proportion of study-defined MR in $\mathrm{AD}$ was $64.0 \%$, in PDD-NOS 29.3\% and overall ASDs 41.2\%. The difference in percentages of children with mental retardation between $\mathrm{AD}$ and PDD-NOS is significant $\left(\chi^{2}=7.670 ; \mathrm{df}=1 ; p<.01\right)$. Boys outnumbered girls by 7.3:1 for AD, and 7.4:1 for PDDNOS. Mean age at intake overall was 5.5 years $(\mathrm{SD}=2.2)$. In children born from 1990-1994 (minimum possible intake age in the first year the clinic was opened: 3-7 years) the mean age at intake was 7.2 years $(\mathrm{SD}=2.1)$. In this group, there was no significant difference in age at intake between children classified as AD and children classified as PDDNOS. For children born 1995-1999 (minimum possible intake age in the first year the clinic was opened: 0-2 years) the mean age at intake was 4.5 years $(\mathrm{SD}=1.5)$. In this group, children classified as AD were significantly younger than children classified with PDD-NOS (3.9 years versus 5.1 years; $t=-2.67 ; \mathrm{df}=41 ; p<.05$ ).

Validation study

The subjects for the validation study included 24 children with a study diagnosis of ASD, and six clinic children 
Table 1 Prevalence per 1,000 births

\begin{tabular}{lcccccc}
\hline Diagnosis & Male & $95 \%$ CI & Female & $95 \%$ CI & Total & $95 \%$ CI \\
\hline AD & 3.3 & $2.0-4.9$ & 0.5 & $0.1-1.4$ & 1.9 & $1.2-2.8$ \\
PDD-NOS & 5.5 & $3.9-7.5$ & 0.8 & $0.3-1.8$ & 3.2 & $2.3-4.3$ \\
Asperger & 0.1 & $0.0-0.8$ & 0.2 & $0.0-0.9$ & 0.2 & $0.0-0.6$ \\
Total ASD & 8.9 & $6.8-11.4$ & 1.4 & $0.6-2.7$ & 5.3 & $4.1-6.7$ \\
\hline
\end{tabular}

diagnosed with other disorders. The independent rater was blinded to diagnostic information prior to the assessment, and assessed 17 of the 24 (70\%) of ASD study cases with the ADOS, while the remaining seven ASD subjects were examined by the first author (IvB). All except one $(95.8 \%$ of 24) of the study diagnoses of ASD were confirmed by the ADOS rating. None of the children diagnosed with other disorders scored for ASD on the ADOS algorithm.

Comparison analysis of cumulative incidence

To compare our data to those described by Powell et al., 2000 [26], we calculated the cumulative incidence in children born in the period January 1, 1996 through December 31, 1998, with intake prior to age five. The cumulative incidence for AD was 2.4 per $1,000(95 \%$ CI 1.1-4.4) and for all ASDs it was 4.5 per $1,000(95 \%$ CI 2.7-7.0). Powell reported a cumulative incidence to age five of 1.6 per $1,000(95 \% \mathrm{CI} 1.1-2.4)$ for $\mathrm{AD}$, and 3.4 per 1,000 (95\% CI 2.5-4.4) for ASDs.

\section{Discussion}

This is the first report of the prevalence of autism spectrum disorders in a Caribbean country. In the Aruba birth years 1990-1999 we found a prevalence for autistic disorder of 1.9 per $1,000(95 \%$ CI $1.2-2.8)$ and for autism spectrum disorders of 5.3 per 1,000 (95\% CI 4.1-6.7). These prevalence estimates should be considered minimum prevalence. Centralized psychiatric services, with excellent coverage and penetration notwithstanding, it is possible that ASD cases have escaped detection within the study period. Children who have left the frame of observation may have been missed cases, but they still were included in the denominator. Higher functioning cases and young children (i.e. year of birth in the late nineties) may not have been referred.

The prevalence found in the present study is in the mid range of estimates reported for similar birth years in studies conducted in the US and Europe, using diverse methodologies [4-8, 27-31]. In Table 3 we present selected autism prevalence studies published from 2000 reporting on children born during the same time period, but diagnosed using varied methodologies.

The Aruban findings are consistent with these reports in two additional respects: the proportion of autism spectrum cases with $\mathrm{AD}(25 / 69=36.2 \%)$, and the proportion of cases with co-morbid mental retardation (41\% ASDs, $64 \% \mathrm{AD}$ ). The sex ratio $(87.0 \%$ males) is also within the range of previous reports $[1,5,7,8,30]$.

The validation study showed a rate of agreement between study diagnosis and ADOS that was higher than expected at $95.8 \%$. For example, Gray et al. [32] showed that the overall agreement between ADOS and clinical diagnosis was .87 (95\% CI .81-.91) in a group of young children (aged 20-55 months). A possible explanation for the higher agreement found in the present study could be the preponderance of $\mathrm{AD}$ ( 16 of 24 subjects), and a higher age distribution; both conditions contribute to a better performance of the ADOS [32, 33].

A recent epidemiological study investigating children aged 3-9 years within different health services in Venezuela [34] yielded a treated prevalence of 1.7 per $1,000(95 \% \mathrm{CI}$ 0.1-2.0) for all ASDs, and 1.1 per 1,000 (95\% CI 1.0-1.4) for autism. There could be various explanations for the markedly lower prevalence estimates in Venezuela, especially for all ASDs, compared to our study. Aruba is

Table 2 Case characteristics

\footnotetext{
${ }^{\text {a }}$ Only one case meeting study criteria of PDD-NOS had the minimum number of two symptoms; all other cases had three or more symptoms
}

\begin{tabular}{llll}
\hline & AD $(n=25)$ & PDD-NOS $(n=42)$ & ASDs $(n=69)$ \\
\hline SYMPTOMS (range) & Mean (sd) & Mean (sd) & Mean (sd) \\
Social (0-4) & $3.8(0.5)$ & $2.7(1.0)$ & $3.1(1.0)$ \\
Communication (0-3) & $2.2(0.7)$ & $1.5(0.8)$ & $1.7(0.9)$ \\
Repetitive Behaviours (0-4) & $2.2(1.1)$ & $0.3(0.6)$ & $1.0(1.2)$ \\
Total Symptoms & $8.2(1.6)$ & $4.5(1.3)^{\mathrm{a}}$ & $5.8(2.3)$ \\
AGE AT INTAKE-years & $4.6(2.3)$ & $6.0(1.9)$ & $5.5(2.2)$ \\
Birth cohort 1990-1999 & $64.0 \%$ & $29.3 \%$ & $41.2 \%$ \\
MENTAL RETARDATION—\% & & & \\
\hline
\end{tabular}


Table 3 A comparison of selected descriptive epidemiology studies of autism

\begin{tabular}{|c|c|c|c|c|c|c|}
\hline & $\begin{array}{l}\text { Powell et al. } \\
2000[26]\end{array}$ & $\begin{array}{l}\text { Baird et al. } \\
2000[27]\end{array}$ & $\begin{array}{l}\text { Bertrand et al. } \\
2001[28]\end{array}$ & $\begin{array}{l}\text { Yeargin-Allsop et al. } \\
2003 \text { [31] }\end{array}$ & $\begin{array}{l}\text { Chakrabarti and } \\
\text { Fombonne } 2005 \text { [29] }\end{array}$ & $\begin{array}{l}\text { van Balkom et al. } \\
\text { Present study }\end{array}$ \\
\hline Population & $16,012^{\mathrm{b}}$ & 16,235 & 8,896 & 289,456 & 10,903 & 13,109 \\
\hline Country & UK & UK & US & US & UK & Aruba \\
\hline Age & $1-4$ years & 7 years & $3-10$ years & $3-10$ years & 4-6 years & 4-13 years \\
\hline Diagnosis $^{\text {a }}$ & $\mathrm{RD}$ & DA & DA & RRD & DA & RRD \\
\hline $\begin{array}{l}\text { Prevalence } \\
\mathrm{AD}\end{array}$ & $1.6^{\mathrm{b}}$ & 3.1 & 4.0 & - & 2.2 & 1.9 \\
\hline $\begin{array}{l}\text { Prevalence } \\
\text { ASDs }\end{array}$ & $3.4^{\mathrm{b}}$ & 5.8 & 6.7 & 3.4 & 5.9 & 5.3 \\
\hline Proportion & $48 \%$ & $53 \%$ & $60 \%$ & - & $38 \%$ & $36 \%$ \\
\hline $\begin{array}{l}\text { AD/all } \\
\text { ASDs }\end{array}$ & $(26 / 54)$ & $(50 / 94)$ & $(36 / 60)$ & & $(24 / 64)$ & $(25 / 69)$ \\
\hline $\begin{array}{l}\text { Male: } \\
\text { Female } \\
\text { ASDs }\end{array}$ & $5.7: 1$ & $7.5: 1$ & $2.8: 1$ & $4: 1$ & 6.1:1 (est) & $6.7: 1$ \\
\hline$\%$ MR: AD & - & $40 \%$ & $58 \%$ & - & $67 \%$ & $64 \%$ \\
\hline $\begin{array}{l}\% \text { MR: } \\
\text { ASDs }\end{array}$ & - & $22 \%$ & $49 \%$ & $64 \%$ & $30 \%$ & $42 \%$ \\
\hline
\end{tabular}

Legenda

${ }^{\mathrm{a}} D A$ Direct Assessment; $R D$ Record Diagnosis; $R R D$ Record Review Diagnosis, ${ }^{\mathrm{b}}$ Cumulative incidence single birth year $\approx$ lifetime prevalence to age 5 for birth year

distinctly different from Venezuela, not only in terms of availability and access to health care, but also with respect to socioeconomic and population characteristics. One explanation is a lower degree of service coverage and penetration in the population compared to the Aruba study, where 6\% of all children born in Aruba between 19901999 were evaluated at the clinic. As mentioned by the authors, a possible lack of awareness of autism and treatment options in the general population could have resulted in underrecognition and lower referral levels for higher functioning ASD cases. Of course, lower prevalence estimates can also reflect lower prevalence in the underlying population.

Because epidemiologic findings with respect to autism spectrum disorders are particularly sensitive to study methodology, the comparison analysis was undertaken in which we drew a direct comparison of cumulative incidence to age five of ASDs in Aruba to that reported by a study conducted in two areas of the West Midlands, UK [26]. In this UK study case ascertainment methods are similar to the present study: children were identified through Child Developmental Centres' treatment records, and similar diagnostic criteria were in use during the period of case detection. Powell reported a cumulative incidence (to age five) of 1.6 per 1,000 for $\mathrm{AD}$, and 3.4 per 1,000 for ASDs. We estimated the cumulative incidence to age five of $\mathrm{AD}$ and ASDs to be 2.4 per $1,000,4.5$ per 1,000 respectively, at the high end of the confidence intervals of the UK study. This difference may be one of methodology.
In the UK study onset was defined as the age at which a definite or probable diagnosis of ASD was first communicated to the child's family, whereas in the present study we used date of first contact. However, it is uncertain whether this slightly higher cumulative incidence could be accounted for by this difference.

The ideal study upon which to base comparisons in prevalence and cumulative incidence across cultures would use the same rigorous methods at all research sites. However, in reality the development of comparable prevalence estimates is hampered by methodological issues, and differences across countries and services.

It was therefore surprising that in spite of stated differences we found, using similar methods, that the prevalence estimate for ASDs previously reported in a narrow range of countries, also pertains in a place as distinctive as Aruba.

Strengths and limitations of the study

The strength of evidence derives from the coverage and penetration of the health care system, the quality of diagnostic services, and the ability to accurately enumerate the population at risk. During the 1990's, health insurance was nearly universal for legal residents, but access to specialty services was limited. From 2001 forward, access to child psychiatry services was effectively universal; over the study period, six percent of all children born in Aruba from 1990 to 1999 were assessed at the clinic. 
The consistency and reliability of diagnosis upon which the prevalence estimates are based also contribute to the strength of the study findings. All children were fully assessed by the clinic psychiatrist and detailed clinical notes were systematically collected and included in charts. Study inclusion was based on standardized chart abstractions; a study diagnosis was assigned in accordance with standardized diagnostic criteria. In an effort to validate study classification of ASD the ADOS was used to examine 35\% $(24 / 69)$ of ASD subjects included in the study. This showed confirmation of study classification in all cases, but one.

Finally, the population of Aruban births 1990-1999 was enumerated based on population registry data. Because virtually all births in Aruba are attended [15], registry data should include the births of all legal Aruban-born children. All children identified with ASDs reported here are legal Aruban residents.

The limitations of this study are those common to record-based prevalence studies, and fall into two principal categories: factors affecting case ascertainment and factors affecting diagnosis. With respect to case ascertainment, a fundamental limitation of record review methodology is that prevalence will only include children who presented for clinical assessment, and who elicited clinical suspicion of falling within the autism spectrum. In past studies, reliance on a single source for identifying cases has yielded low estimates [31]. In this Aruban context, diagnostic assessment and treatment is centralized in the first and only child psychiatry service on the island; competing diagnostic services do not exist.

Other ascertainment effects are specific to segments of the cohort, and are only relevant in the context of the main prevalence analysis. Some cases among children born in the earliest birth years and in the latest birth years may not have been referred to the child psychiatric service. Because the clinic opened in 1997 , it is possible that cases born in the early 1990s emigrated in search of services prior to the clinic's opening and thereby escaped detection. It is also possible that higher functioning cases born in the late 1990s have not attained a sufficient age for referral and are therefore underrepresented in this study.

Another ascertainment issue may be relevant to both the main prevalence analysis and the comparison analysis, that is that ascertainment of children with prominent comorbidities, especially mental retardation, may also have been limited. Interviews with directors of Aruban schools and day-care programs for the disabled concerning the diagnostic distribution in their institutions, however, lead us to believe that few lower functioning children were overlooked.

With respect to diagnosis, as in any record-based study, the findings are limited by the absence of in-person standardized research interviews and assessments of every study classified case. Due to restraints in time and finances only 24 of the 69 children (almost 35\%) with a study classification of ASD were assessed with the ADOS. One study classified ASD case was not confirmed by the ADOS rating.

A potential for misdiagnosis specific to this population arises from the multilingual environment. It is possible that some children with late language development and behavioral disturbances (e.g., ADHD) present with ASD-like profiles at some point in their development.

In conclusion, it is clear that interest in the distribution of ASDs is intensifying, as concern over possible environmental contributions to the occurrence of these disorders continues to grow [3]. Standardizing future research methodology would permit geographic cross-cultural comparisons. Finding areas of high and low contrast will also motivate additional international epidemiologic investigations. In addition, each epidemiologic study will contribute to local appreciation of the magnitude of ASDs impact on local public health resources and services.

This study shows that the prevalence of ASDs in Aruba is similar to previous prevalence reports from a narrow range of developed countries. We hesitate to draw inferences about the causes of autism based on findings from a single setting. However, as studies in diverse settings accumulate, we believe that the emerging picture will provide important clues to some causes and help to rule out others. The significance of these findings for Aruba is clear. Aruba joins the developed world in needing to respond with services and care for a significant number of seriously disabled individuals.

Acknowledgements We gratefully acknowledge Prof. Dr. I.A. van Berckelaer-Onnes (Netherlands) and Inge Boutier (Curaçao) for their diagnostic help. We would also like to thank Sophie Hennekam, Krysten Ho- Kang-You, Miriam van Beek and Eliana de Cuba for their assistance with the many organizational aspects of the study.

Declaration of interest None.

\section{References}

1. Fombonne E. Epidemiological surveys of autism and other pervasive developmental disorders: an update. J Autism Dev Disord. 2003;33:365-82.

2. Fombonne E. Epidemiology of pervasive developmental disorders. Pediatr Res. 2009.

3. Kolevzon A, Gross R, Reichenberg A. Prenatal and perinatal risk factors for autism: a review and integration of findings. Arch Pediatr Adolesc Med. 2007;161:326-33.

4. Baird G, Simonoff E, Pickles A, Chandler S, Loucas T, Meldrum D, et al. Prevalence of disorders of the autism spectrum in a population cohort of children in South Thames: the Special Needs and Autism Project (SNAP). Lancet. 2006;368:210-5.

5. Fombonne E, Zakarian R, Bennett A, Meng L, McLean-Heywood D. Pervasive developmental disorders in Montreal, Quebec, 
Canada: prevalence and links with immunizations. Pediatrics. 2006;118:e139-50.

6. Petersen DJ, Bilenberg N, Hoerder K, Gillberg C. The population prevalence of child psychiatric disorders in Danish 8- to 9-yearold children. Eur Child Adolesc Psychiatry. 2006;15:71-8.

7. Centers for Disease Control and Prevention. Prevalence of autism spectrum disorders-autism and developmental disabilities monitoring network, six sites, United States, 2000. MMWR Surveill Summ. 2007;56:1-11.

8. Centers for Disease Control and Prevention. Prevalence of autism spectrum disorders-autism and developmental disabilities monitoring network, 14 sites, United States, 2002. MMWR Surveill Summ. 2007;56:12-28.

9. Ellefsen A, Kampmann H, Billstedt E, Gillberg IC, Gillberg C. Autism in the Faroe Islands: an epidemiological study. J Autism Dev Disord. 2007;37:437-44.

10. Ghanizadeh A. A preliminary study on screening prevalence of pervasive developmental disorder in schoolchildren in Iran. J Autism Dev Disord. 2008;38:759-63.

11. Oliveira G, Ataide A, Marques C, Miguel TS, Coutinho AM, Mota-Vieira L, et al. Epidemiology of autism spectrum disorder in Portugal: prevalence, clinical characterization, and medical conditions. Dev Med Child Neurol. 2007;49:726-33.

12. Hoek HW, van Harten PN, van Hoeken D, Susser E. Lack of relation between culture and anorexia nervosa-Results of an incidence study on Curaçao. N Engl J Med. 1998;338:1231-2.

13. Hoek HW, van Harten PN, Hermans KME, Katzman MA, Matroos GE, Susser ES. The incidence of anorexia nervosa on Curaçao. Am J Psychiatry. 2005;162:748-52.

14. Toro-Labrador G, Wever OR, Martinez-Cruzado JC. Mitochondrial DNA analysis in Aruba: strong maternal ancestry of closely related Amerindians and implications for the peopling of Northwestern Venezuela. Caribb J Sci. 2003;39:11-22.

15. Pan American Health Organization. Health in the Americas. 2002 Edition ed. Washington, DC: PAHO; 2002.

16. United States Census Bureau. Global Population Profile 2002, Appendix A, tables A-11, A-12; 2002. Report No.: http://www. census.gov/ipc/www/wp02.html.

17. World Health Organization (WHO). WHO Mortality Database; 2005. Report No.: http://www.who.int/healthinfo/morttables/en/ index.html.

18. Van Balkom ID, Hagendoorn J, De Pater JM, Hennekam RC. Partial monosomy $8 \mathrm{p}$ and partial trisomy $8 \mathrm{p}$ with moderate mental retardation. Genet Couns. 1992;3:83-9.

19. Van Balkom ID, Quartel S, Hennekam RC. Mental retardation, "coarse" face, and hyperbreathing: confirmation of the PittHopkins syndrome. Am J Med Genet. 1998;75:273-6.

20. Van Balkom ID, Alders M, Allanson J, Bellini C, Frank U, De Jong $\mathrm{G}$, et al. Lymphedema-lymphangiectasia-mental retardation (Hennekam) syndrome: a review. Am J Med Genet. 2002; 112:412-21.
21. Charman T. What does the term 'working diagnosis' mean? J Autism Dev Disord. 2005;35:539-40.

22. Lord C, Rutter M, Goode S, Heemsbergen J, Jordan H, Mawhood L, et al. Autism diagnostic observation schedule: a standardized observation of communicative and social behavior. J Autism Dev Disord. 1989;19:185-212.

23. Lord C, Risi S, Lambrecht L, Cook EH Jr, Leventhal BL, DiLavore PC, et al. The autism diagnostic observation schedulegeneric: a standard measure of social and communication deficits associated with the spectrum of autism. J Autism Dev Disord. 2000;30:205-23.

24. Central Bureau of Statistics (CBS). Statistical Yearbook 2002. Oranjestad, Aruba: CBS; 2003.

25. STATA. Stata statistical software for Professionals: Release 8.0. College Station, TX: StataCorp.; 2003.

26. Powell JE, Edwards A, Edwards M, Pandit BS, Sungum-Paliwal SR, Whitehouse W. Changes in the incidence of childhood autism and other autistic spectrum disorders in preschool children from two areas of the West Midlands, UK. Dev Med Child Neurol. 2000;42:624-8.

27. Baird G, Charman T, Baron-Cohen S, Cox A, Swettenham J, Wheelwright $\mathrm{S}$, et al. A screening instrument for autism at 18 months of age: a 6-year follow-up study. J Am Acad Child Adolesc Psych. 2000;39:694-702.

28. Bertrand J, Mars A, Boyle C, Bove F, Yeargin-Allsopp M, Decoufle P. Prevalence of autism in a United States population: the Brick Township, New Jersey, investigation. Pediatrics. 2001;108:1155-61.

29. Chakrabarti S, Fombonne E. Pervasive developmental disorders in preschool children: confirmation of high prevalence. Am J Psychiatry. 2005;162:1133-41.

30. Gillberg C, Cederlund M, Lamberg K, Zeijlon L. Brief report: "the autism epidemic". The registered prevalence of autism in a Swedish urban area. J Autism Dev Disord. 2006;36:429-35.

31. Yeargin-Allsopp M, Rice C, Karapurkar T, Doernberg N, Boyle C, Murphy C. Prevalence of autism in a US metropolitan area. JAMA. 2003;289:49-55.

32. Gray KM, Tonge BJ, Sweeney DJ. Using the Autism Diagnostic Interview-Revised and the Autism Diagnostic Observation Schedule with young children with developmental delay: evaluating diagnostic validity. J Autism Dev Disord. 2008;38:657-67.

33. De Bildt A, Sytema S, Ketelaars C, Kraijer D, Mulder E, Volkmar $\mathrm{F}$, et al. Interrelationship between Autism Diagnostic Observation Schedule-Generic (ADOS-G), Autism Diagnostic InterviewRevised (ADI-R), and the Diagnostic and Statistical Manual of Mental Disorders (DSM-IV-TR) classification in children and adolescents with mental retardation. J Autism Dev Disord. 2004;34:129-37.

34. Montiel-Nava C, Pena JA. Epidemiological findings of pervasive developmental disorders in a Venezuelan study. Autism. 2008;12:191-202. 